\title{
On the quantum field theory of the gravitational interactions
}

\author{
Damiano Anselmi \\ Dipartimento di Fisica "Enrico Fermi", Università di Pisa, \\ Largo B. Pontecorvo 3, 56127 Pisa, Italy \\ INFN, Sezione di Pisa, \\ Largo B. Pontecorvo 3, 56127 Pisa, Italy \\ E-mail: damiano.anselmi@unipi.it
}

ABSTRACT: We study the main options for a unitary and renormalizable, local quantum field theory of the gravitational interactions. The first model is a Lee-Wick superrenormalizable higher-derivative gravity, formulated as a nonanalytically Wick rotated Euclidean theory. We show that, under certain conditions, the $S$ matrix is unitary when the cosmological constant vanishes. The model is the simplest of its class. However, infinitely many similar options are allowed, which raises the issue of uniqueness. To deal with this problem, we propose a new quantization prescription, by doubling the unphysical poles of the higher-derivative propagators and turning them into Lee-Wick poles. The Lagrangian of the simplest theory of quantum gravity based on this idea is the linear combination of $R, R_{\mu \nu} R^{\mu \nu}, R^{2}$ and the cosmological term. Only the graviton propagates in the cutting equations and, when the cosmological constant vanishes, the $S$ matrix is unitary. The theory satisfies the locality of counterterms and is renormalizable by power counting. It is unique in the sense that it is the only one with a dimensionless gauge coupling.

Keywords: Models of Quantum Gravity, Beyond Standard Model, Renormalization Regularization and Renormalons

ArXiv EPrint: 1704.07728 


\section{Contents}

1 Introduction 1

$\begin{array}{llr}2 & \text { Superrenormalizable quantum gravity } & 3\end{array}$

2.1 Renormalization 4

$\begin{array}{lll}2.2 & \text { Unitarity } & 6\end{array}$

3 Coupling to matter $\quad 9$

$\begin{array}{lll}4 & \text { The problem of uniqueness } & 11\end{array}$

$5 \quad$ Fake degrees of freedom $\quad 12$

6 Quantum gravity with a dimensionless gauge coupling $\quad 14$

$\begin{array}{lll}7 & \text { Conclusions } & 16\end{array}$

\section{Introduction}

The problem of quantum gravity is the compatibility between renormalizability and unitarity. It is well known that the Hilbert-Einstein action is not renormalizable by power counting [1-4]. However, if we include the infinitely many counterterms it generates, multiplied by independent couplings, it is perturbatively unitary [5]. An option to improve the ultraviolet behavior of the loop integrals is to add quadratic terms with higher derivatives. It is then possible to build higher-derivative theories of quantum gravity that are renormalizable with finitely many couplings [6-9]. However, such theories are not unitary, at least if they are formulated in the usual ways.

Higher-derivative theories must be formulated properly, because they are less trivial than one would naively expect. For example, if they are defined directly in Minkowski spacetime, i.e. by integrating the loop energies along the real axis of the complex energy plane, they generate nonlocal, non-Hermitian divergences when the free propagators have complex poles [10], which makes them unacceptable from the mathematical point of view. On the other hand, the Wick rotation from Euclidean space is obstructed when the free propagators have poles in the first or third quadrants of the complex energy plane. The obstruction can actually be overcome by a nonanalytic procedure, which leads to a new formulation [11] of an interesting subclass of higher-derivative theories, the Lee-Wick (LW) models $[12,13]$.

Viewed as nonanalytically Wick rotated Euclidean theories, such models are perturbatively unitary [14]. Moreover, the new formulation is intrinsically equipped with all that is needed to define the physical amplitudes properly, with no need of ad hoc prescriptions. 
The complex energy hyperplane is divided into disjoint regions $\mathcal{A}_{i}$ of analyticity, which can be connected to one another by a well defined, but nonanalytic procedure. It is necessary to work in suitable subsets $\mathcal{O}_{i}$ of the regions $\mathcal{A}_{i}$, in a generic Lorentz frame, and analytically continue the results from $\mathcal{O}_{i}$ to $\mathcal{A}_{i}$ at the end. Finally, the nonanalytic behaviors of the physical amplitudes suggest ways that may facilitate the experimental measurements of the key parameters of the models.

Old formulations of the Lee-Wick models were based on ad hoc prescriptions, the best known one being the CLOP prescription of ref. [15]. ${ }^{1}$ Often, such approaches are unambiguous in some loop diagrams, but ambiguous in others, and do not admit a clear formulation at the Lagrangian level. In ref. [11] it has been shown that they may give ambiguous results already at one loop.

In this paper, we investigate the main options for quantum gravity that are offered by the nonanalytic Wick rotation of Euclidean higher-derivative theories, combined with extra tools that we introduce anew. We begin with the superrenormalizable Lee-Wick models, which are unitary when the cosmological constant vanishes. We investigate the simplest representative of this class of models in detail and show that in various cases a vanishing cosmological constant is consistent with the renormalization group, before and after the coupling to matter. However, the theories with similar properties are infinitely many, which raises the issue of uniqueness. A principle of maximum simplicity could be used to single out the model studied here, but the principle itself would have to be justified in its turn. For this reason, it is worth to move further on, in the search for a unique theory of quantum gravity. We identify a candidate in a model whose Lagrangian contains the Hilbert-Einstein term, the cosmological term, $R_{\mu \nu} R^{\mu \nu}$ and $R^{2}$. We formulate it by means of an additional trick, which consists of doubling the ghost poles of the free propagators and treating the doubled versions as Lee-Wick poles. The perturbative unitarity of the model then follows from the one of its Lee-Wick parent theory. The uniqueness of this options relies on the fact that it is the only one whose gauge coupling is dimensionless (according to the power counting of the high-energy limit).

The LW models have been studied in QED [13], the standard model [17-20] and grand unified theories [21, 22], besides quantum gravity [23-27]. Although the CLOP or other ad hoc prescriptions have been advocated in such investigations, some conclusions may survive once those prescriptions are removed in favor of the formulation of ref. [11].

We recall other options to make sense of quantum gravity that can be found in the literature. A well known idea is asymptotic safety [28]. If there exists an ultraviolet interacting fixed point with a finite dimensional critical surface, then it is possible to reduce the free parameters of quantum gravity to a finite number, by demanding that the theory lie on the critical surface at high energies. The weakness of this approach is that it is nonperturbative. Nevertheless, truncations and consistency checks can provide evidence that ultraviolet fixed points may indeed exist and have good critical surfaces [29-32].

Nonlocal theories of quantum gravity have also been explored [33-39]. Some theories of this class are claimed to have a simple, local renormalization [33-37]. This may be true

\footnotetext{
${ }^{1}$ See $[16]$ for explicit calculations in this approach.
} 
if they are defined in Euclidean space, but the results of [10] suggest that if they are defined in Minkowski spacetime, they generate nonlocal divergences that cannot be removed by any standard procedures. On the other hand, it is hard to Wick rotate such nonlocal Euclidean theories, because their free propagators contain nonpolynomial functions that have extremely involved behaviors at infinity in the first and third quadrants of the complex energy plane. Finally, the usual proofs of perturbative unitarity [40-43] do not extend to nonlocal theories straightforwardly [5].

Other possibilities to make sense of higher-derivative theories have been explored. One is that the unphysical degrees of freedom, even if present, might be unobservable if the renormalization group keeps their masses always above the running energy [44, 45]. Another possibility is that the unphysical degrees of freedom might be a blunder due to the expansion around the wrong vacuum.

We also recall that it is possible to treat quantum gravity as a low energy effective field theory with infinitely many couplings. In principle, this approach can even lead to physical predictions beyond the low-energy regime, if we identify physical quantities that just depend on a finite subset of parameters. For example, organizing the Lagrangian in a convenient way [46], it can be proved that the Friedmann-Lemaître-Robertson-Walker (FLRW) metrics are exact solutions of the complete field equations in arbitrary dimensions with a homogeneous and isotropic matter distribution (after a perturbative field redefinition of the metric tensor).

The paper is organized as follows. In section 2 we study the simplest superrenormalizable model of quantum gravity and work out the conditions under which it is unitary. In section 3 we extend the analysis to the coupling to matter. In section 4 we address the uniqueness problem. In section 5 we introduce the concept of fake degree of freedom. By turning ghosts into fakes, in section 6 we build the unique model of quantum gravity that has a dimensionless gauge coupling and show that it is unitary up to "anomalous" effects due to the cosmological constant. Section 7 contains our conclusions.

\section{Superrenormalizable quantum gravity}

The first option that we consider is a superrenormalizable higher-derivative gravity, formulated by nonanalytically Wick rotating its Euclidean version. We focus on the simplest representative of this class. Up to total derivatives, its most general Lagrangian $\mathcal{L}_{\mathrm{QG}}$ is given by

$$
\begin{aligned}
-2 \kappa^{2} \mu^{\varepsilon} \frac{\mathcal{L}_{\mathrm{QG}}}{\sqrt{-g}}= & 2 \lambda_{C} M^{2}+\zeta R-\frac{\gamma}{M^{2}} R_{\mu \nu} R^{\mu \nu}+\frac{1}{2 M^{2}}(\gamma-\eta) R^{2} \\
& -\frac{1}{M^{4}}\left(D_{\rho} R_{\mu \nu}\right)\left(D^{\rho} R^{\mu \nu}\right)+\frac{1}{2 M^{4}}(1-\xi)\left(D_{\rho} R\right)\left(D^{\rho} R\right) \\
& +\frac{1}{M^{4}}\left(\alpha_{1} R_{\mu \nu} R^{\mu \rho} R_{\rho}^{\nu}+\alpha_{2} R R_{\mu \nu} R^{\mu \nu}+\alpha_{3} R^{3}+\alpha_{4} R R_{\mu \nu \rho \sigma} R^{\mu \nu \rho \sigma}\right. \\
& \left.+\alpha_{5} R_{\mu \nu \rho \sigma} R^{\mu \rho} R^{\nu \sigma}+\alpha_{6} R_{\mu \nu \rho \sigma} R^{\rho \sigma \alpha \beta} R_{\alpha \beta}^{\mu \nu}\right),
\end{aligned}
$$


where $\lambda_{C}, \zeta, \gamma, \eta, \xi, \alpha_{1}, \cdots, \alpha_{6}$ are dimensionless constants, $\kappa$ has dimension -1 in units of mass and $M$ is the Lee-Wick mass scale. The last two lines contain a convenient basis for the six independent scalars that can be built with three Riemann tensors.

We expand the metric tensor $g_{\mu \nu}$ around the Galilean metric $\eta_{\mu \nu}=\operatorname{diag}(1,-1,-1,-1)$ by writing

$$
g_{\mu \nu}=\eta_{\mu \nu}+2 \kappa h_{\mu \nu},
$$

where $h_{\mu \nu}$ is the quantum fluctuation. After the expansion around flat space, we raise and lower the indices by means of the Galilean metric. We further define $h \equiv h_{\mu}^{\mu}$. It is convenient to choose a gauge-fixing function that is linear in the fluctuation $h_{\mu \nu}$, such as the De Donder function

$$
\mathcal{G}_{\mu}(g)=\eta^{\nu \rho} \partial_{\rho} g_{\mu \nu}-\frac{1}{2} \eta^{\nu \rho} \partial_{\mu} g_{\nu \rho}
$$

We complete the gauge-fixing following the steps of ref. [10], so as to obtain the gauge-fixed Lagrangian

$$
\begin{aligned}
\mathcal{L}_{\mathrm{gf}}= & \mathcal{L}_{\mathrm{QG}}+\frac{1}{4 \kappa^{2}} \mathcal{G}^{\mu}\left(\zeta-\gamma \frac{\square}{M^{2}}+\frac{\square^{2}}{M^{4}}\right) \mathcal{G}_{\mu} \\
& +\bar{C}^{\mu}\left(\zeta-\gamma \frac{\square}{M^{2}}+\frac{\square^{2}}{M^{4}}\right)\left[\square C_{\mu}-\left(2 \delta_{\mu}^{\rho} \eta^{\nu \sigma} \partial_{\nu}-\eta^{\rho \sigma} \partial_{\mu}\right) \Gamma_{\rho \sigma}^{\alpha} C_{\alpha}\right],
\end{aligned}
$$

where $\square=\eta^{\mu \nu} \partial_{\mu} \partial_{\nu}$ is the flat-space D'Alembertian.

We begin by studying the renormalization of the theory and then discuss the conditions under which it is perturbatively unitary.

\subsection{Renormalization}

It is easy to see that the renormalization of a LW theory, formulated as the nonanalytic Wick rotation of its Euclidean version, coincides with the renormalization of its Euclidean version. Consider a Feynman diagram and integrate the loop energies by means of the residue theorem, as usual. We recall $[11,14]$ that the nonanalytic behavior of the Wick rotation is due to the pinching of LW poles and that the LW pinching conditions equate a linear combination $p^{0}$ of the external energies to a sum of frequencies $\omega_{i}(\mathbf{k}, \mathbf{p})$, where $\mathbf{k}$ denotes the loop space momenta. Now, the ultraviolet divergences are studied by keeping the external momenta $p$ fixed and letting $\mathbf{k}$ tend to infinity. In such a limit the LW pinching conditions have no solutions, because the frequencies $\omega_{i}$ grow linearly, but their sum is fixed. For this reason, the LW pinching does not affect the renormalization of the theory, which allows us to study the counterterms of $\mathcal{L}_{\mathrm{QG}}$ with the usual techniques.

By power counting, the counterterms have at most dimension four. Using the dimensional regularization, we organize them as

$$
\frac{\mathcal{L}_{\text {count }}}{\sqrt{-g}}=\frac{1}{(4 \pi)^{2} \varepsilon}\left[2 a_{C} M^{4}+a_{\zeta} M^{2} R-a_{\gamma} R_{\mu \nu} R^{\mu \nu}+\frac{1}{2}\left(a_{\gamma}-a_{\eta}\right) R^{2}\right]
$$

where $\varepsilon=4-D, D$ being the continued spacetime dimension. It is easy to see that the counterterms proportional to the cosmological constant are present up to three loops, those proportional to the Hilbert-Einstein term are present up to two loops and the counterterms 
proportional to $R_{\mu \nu} R^{\mu \nu}$ and $R^{2}$ are just present at one loop. The parameters $\xi$ and $\alpha_{i}$ do not run.

It is convenient to introduce the "fine structure constant of quantum gravity"

$$
\alpha_{\mathrm{QG}}=\frac{\kappa^{2} M^{2}}{4 \pi} \text {. }
$$

To minimize the number of $\pi \mathrm{s}$ in the formulas below, we also introduce the constant

$$
\bar{\alpha}=\frac{\kappa^{2} M^{2}}{(4 \pi)^{2}}=\frac{\alpha_{\mathrm{QG}}}{4 \pi} .
$$

The structure of the coefficients that appear in formula (2.2) is

$$
a_{C}=a_{C}^{(1)}+u \gamma \bar{\alpha}+v \eta \bar{\alpha}+w \bar{\alpha}^{2}, \quad a_{\zeta}=a_{\zeta}^{(1)}+z \bar{\alpha} \quad a_{\gamma}=a_{\gamma}^{(1)}, \quad a_{\eta}=a_{\eta}^{(1)},
$$

where the superscript "(1)" denotes the one-loop values, while

$$
u=u_{1}+\frac{u_{2}}{\varepsilon}, \quad v=v_{1}+\frac{v_{2}}{\varepsilon}, \quad w=w_{1}+\frac{w_{2}}{\varepsilon}+\frac{w_{3}}{\varepsilon^{2}}, \quad z=z_{1}+\frac{z_{2}}{\varepsilon},
$$

and the coefficients $u_{i}, v_{i}, w_{i}, z_{i}$, are functions of the parameters $\alpha_{i}$ and $\xi$. As usual, the renormalization group relates the coefficients $u_{2}, v_{2}, w_{2}, w_{3}$ and $z_{2}$ of the double and triple $\varepsilon$ poles to the coefficients of the simple poles $u_{1}, v_{1}, w_{1}$ and $z_{1}$.

The bare parameters read

$$
\begin{aligned}
\lambda_{C \mathrm{~B}} & =\lambda_{C}-\frac{2}{\varepsilon} a_{C} \bar{\alpha}, & \zeta_{\mathrm{B}} & =\zeta-\frac{2}{\varepsilon} a_{\zeta} \bar{\alpha}, \quad \gamma_{\mathrm{B}}=\gamma-\frac{2}{\varepsilon} a_{\gamma} \bar{\alpha}, \\
\eta_{\mathrm{B}} & =\eta-\frac{2}{\varepsilon} a_{\eta} \bar{\alpha}, & \bar{\alpha}_{\mathrm{B}} & =\bar{\alpha} \mu^{\varepsilon} .
\end{aligned}
$$

From these expressions, we find the beta functions

$$
\begin{aligned}
& \beta_{\zeta}=-2 a_{\zeta}^{(1)} \bar{\alpha}-4 z_{1} \bar{\alpha}^{2}, \quad \beta_{\gamma}=-2 a_{\gamma} \bar{\alpha}, \quad \beta_{\eta}=-2 a_{\eta} \bar{\alpha}, \\
& \beta_{C}=-2 a_{C}^{(1)} \bar{\alpha}-2\left(2 u_{1} \gamma+2 v_{1} \eta+3 w_{1} \bar{\alpha}\right) \bar{\alpha}^{2}, \quad \beta_{\mathrm{QG}}=0,
\end{aligned}
$$

where $\beta_{C}$ is the beta function of $\lambda_{C}$ and $\beta_{\mathrm{QG}}$ is the beta function of $\alpha_{\mathrm{QG}}$. The cancelation of the divergences inside the beta functions gives $u_{2}, v_{2}, w_{2}, w_{3}$ and $z_{2}$.

We have computed the one-loop counterterms in the most general case. However, due to their involved expressions, we just report them in a simplified case that is enough for our purposes, ${ }^{2}$ i.e. at $\xi=\alpha_{6}=0$, where we find

$$
\begin{aligned}
a_{C}^{(1)}= & \frac{3}{4}\left(4 \zeta-2 \gamma^{2}+2 \eta \gamma-3 \eta^{2}\right), \quad a_{\zeta}^{(1)}=\frac{1}{4} \gamma \tau+\frac{1}{2} \eta \sigma, \\
240 a_{\gamma}= & 756-1080 \alpha_{1}+360 \alpha_{1}^{2}+480 \alpha_{2}-480 \alpha_{1} \alpha_{2}-640 \alpha_{2}^{2}+960 \alpha_{4}-960 \alpha_{1} \alpha_{4} \\
& -2560 \alpha_{2} \alpha_{4}-5440 \alpha_{4}^{2}+1940 \alpha_{5}-1080 \alpha_{1} \alpha_{5}-1280 \alpha_{2} \alpha_{5}-3040 \alpha_{4} \alpha_{5}-225 \alpha_{5}^{2}, \\
240 a_{\eta}= & -508-1440 \alpha_{1}+1395 \alpha_{1}^{2}-2400 \alpha_{2}+5160 \alpha_{1} \alpha_{2}+6880 \alpha_{2}^{2}-8640 \alpha_{3}+12960 \alpha_{1} \alpha_{3} \\
& +43200 \alpha_{2} \alpha_{3}+77760 \alpha_{3}^{2}-1920 \alpha_{4}+9600 \alpha_{1} \alpha_{4}+17920 \alpha_{2} \alpha_{4}+34560 \alpha_{3} \alpha_{4} \\
& +20800 \alpha_{4}^{2}+180 \alpha_{5}+1170 \alpha_{1} \alpha_{5}+3560 \alpha_{2} \alpha_{5}+11520 \alpha_{3} \alpha_{5}+5120 \alpha_{4} \alpha_{5}+520 \alpha_{5}^{2},
\end{aligned}
$$

\footnotetext{
${ }^{2}$ The full beta functions can be downloaded in various formats from the website http://renormalization.com at the link http://renormalization.com/Math/QG.
} 
where

$$
\tau=8+9 \alpha_{1}-72 \alpha_{3}+64 \alpha_{4}+3 \alpha_{5}, \quad \sigma=-12+9 \alpha_{1}+30 \alpha_{2}+108 \alpha_{3}+24 \alpha_{4}+8 \alpha_{5} .
$$

\subsection{Unitarity}

To have a correct low-energy limit, we must assume $\zeta>0$. Other important conditions on the parameters of $\mathcal{L}_{\mathrm{QG}}$ follow from unitarity, i.e. the very requirement that the theory is a Lee-Wick model, which we then formulate by nonanalytically Wick rotating its Euclidean version.

First, the extra poles of the free propagators must not be located on the real axis, but lie symmetrically with respect to it, as in

$$
i S(p)=\frac{i}{p^{2}-m^{2}+i \epsilon} \frac{M^{4}}{\left(p^{2}-\mu^{2}\right)^{2}+M^{4}} .
$$

Second, we need to have an identically vanishing cosmological constant. Indeed, when the cosmological constant $\lambda_{C}$ is nonvanishing, flat space is not a solution of the field equations in the absence of matter. The proof of unitarity cannot be carried out to the very end in that case, because it is not known how to build conventional asymptotic states and a consistent scattering matrix $S$ in nonflat spaces [such as (anti) de Sitter space], although alternative approaches have been attempted [47, 48].

We report the graviton propagator in two steps. In the relatively simple case $\eta=\xi=0$, we have

$$
\left\langle h_{\mu \nu}(p) h_{\rho \sigma}(-p)\right\rangle_{\eta=\xi=0}^{\text {free }}=\frac{i M^{4}}{2} \frac{\eta_{\mu \rho} \eta_{\nu \sigma}+\eta_{\mu \sigma} \eta_{\nu \rho}-\eta_{\mu \nu} \eta_{\rho \sigma}}{P\left(1, \gamma, \zeta, 2 \lambda_{C}\right)}
$$

where

$$
P(a, b, c, d) \equiv a\left(p^{2}\right)^{3}+b M^{2}\left(p^{2}\right)^{2}+c M^{4} p^{2}+d M^{6} .
$$

At nonvanishing $\eta$ and $\xi$ the free propagator reads

$$
\begin{aligned}
\left\langle h_{\mu \nu}(p) h_{\rho \sigma}(-p)\right\rangle^{\text {free }}= & \left\langle h_{\mu \nu}(p) h_{\rho \sigma}(-p)\right\rangle_{\eta=\xi=0}^{\text {free }} \\
& -\frac{i M^{4}\left(\eta M^{2}+\xi p^{2}\right)}{2 P\left(1, \gamma, \zeta, 2 \lambda_{C}\right)} \frac{\left(p^{2} \eta_{\mu \nu}+2 p_{\mu} p_{\nu}\right)\left(p^{2} \eta_{\rho \sigma}+2 p_{\rho} p_{\sigma}\right)}{P\left(1-3 \xi, \gamma-3 \eta, \zeta, 2 \lambda_{C}\right)} .
\end{aligned}
$$

As said, we must ensure that the denominators have the correct LW form at $\lambda_{C}=0$, as in (2.3). This happens (with $m=0$ ), if

$$
\gamma^{2}<4 \zeta, \quad(\gamma-3 \eta)^{2}<4 \zeta(1-3 \xi), \quad \gamma<0, \quad \gamma<3 \eta
$$

We must also ensure that the renormalization group is compatible with an identically vanishing cosmological constant. We begin by studying this issue in the simple case $\lambda_{C}=$ $\eta=\xi=\alpha_{6}=0$. Some unitarity bounds (2.6) become identical, so we just have

$$
\gamma^{2}<4 \zeta, \quad \gamma<0
$$


While the conditions $\xi=\alpha_{6}=0$ are preserved by renormalization, because $\xi$ and $\alpha_{6}$ do not run, the conditions $\lambda_{C}=\eta=0$ must be accompanied, for consistency with renormalization group invariance, by

$$
\beta_{C}=\beta_{\eta}=0
$$

The condition $\beta_{\eta}=0$ is relatively easy to solve (see below) and constrains the parameters $\alpha_{i}$. The condition $\beta_{C}=0$ gives

$$
3\left(2 \zeta-\gamma^{2}\right)+2\left(2 u_{1} \gamma+3 w_{1} \bar{\alpha}\right) \bar{\alpha}=0
$$

and is also easy to solve, because it just gives $\zeta$ as a function of the other parameters.

Renormalitazion group invariance demands that the beta function of (2.9) be also zero, which gives

$$
3 \gamma\left(4 a_{\gamma}-\tau\right)=8\left(3 z_{1}+u_{1} a_{\gamma}\right) \bar{\alpha} .
$$

In turn, the beta function of this relation must vanish, which implies

$$
\left(4 a_{\gamma}-\tau\right) a_{\gamma}=0
$$

The beta function of this relation is identically zero, so the list of consistency conditions stops here.

Equation (2.11) implies either $a_{\gamma}=0$ or $a_{\gamma}=-\tau / 4$. The second possibility implies that the right-hand side of (2.10) is zero. However, this condition requires knowledge about the two-loop renormalization of the theory, because it involves $u_{1}$ and $z_{1}$. For this reason, we solve (2.11) by setting $a_{\gamma}=0$, to work out solutions that just need the renormalization at one loop.

Now, $a_{\gamma}=0$ implies $\beta_{\gamma}=0$ and ensures that $\gamma$ is a number that we can choose at will. Equations (2.10) then gives $\beta_{\zeta}=0$, which leads to $\lambda_{C}=\eta=\beta_{C}=\beta_{\eta}=\beta_{\gamma}=\beta_{\zeta}=0$, which means that the Lagrangian (2.1) must be completely finite.

Summarizing, to enforce finiteness, we must solve the system of equations

$$
\zeta=\frac{\gamma^{2}}{2}-\frac{1}{3}\left(2 u_{1} \gamma+3 w_{1} \bar{\alpha}\right) \bar{\alpha}, \quad a_{\gamma}=a_{\eta}=0, \quad \tau \gamma=-8 z_{1} \bar{\alpha} .
$$

The first condition just gives $\zeta$ in terms of the other constants. Since $\zeta$ must be nonvanishing at $\bar{\alpha}=0$, because it multiplies the Hilbert-Einstein term, the same must be true of $\gamma$.

The easiest way to show that solutions do exist is to work them out at $\bar{\alpha}=0$ and check that they can be extended perturbatively to arbitrary $\bar{\alpha}$. Then, at $\bar{\alpha}=0$ we must take

$$
\zeta=\frac{\gamma^{2}}{2}
$$

which is compatible with the unitarity bound (2.7). At this point, we must solve the system

$$
a_{\gamma}=a_{\eta}=\tau=0,
$$


for the parameters $\alpha_{i}$. The condition $\tau=0$ is linear in $\alpha_{i}$ and can be easily solved for one of such parameters. Inserting the solution into the conditions $a_{\gamma}=a_{\eta}=0$, we get two coupled quadratic equations in five unknowns. Acceptable solutions are easy to find algebraically, after setting three parameters $\alpha_{i}$ to zero. For example, we set $\alpha_{4}=\alpha_{5}=\alpha_{6}=0$ and solve $\tau=0$ for $\alpha_{3}$. So doing, we obtain a system of two quadratic equations $e_{1}\left(\alpha_{1}, \alpha_{2}\right)=$ $e_{2}\left(\alpha_{1}, \alpha_{2}\right)=0$ in the two unknowns $\alpha_{1}, \alpha_{2}$. The four real solutions at $\alpha_{4}=\alpha_{5}=\alpha_{6}=0$ are

$$
\begin{aligned}
\left(\alpha_{1}, \alpha_{2}\right)= & (4.51163 \ldots,-3.91524 \ldots), \quad(2.89114 \ldots,-1.93684 \ldots), \\
& (0.800169 \ldots,-0.368609 \ldots), \quad(0.197062 \ldots,-0.679314 \ldots),
\end{aligned}
$$

while $\alpha_{3}=\left(8+9 \alpha_{1}\right) / 72$. All such solutions can be extended perturbatively to nonvanishing $\bar{\alpha}$, because the matrices of the derivatives $\partial e_{i} / \partial \alpha_{j}, i, j \leqslant 2$, are nonsingular on (2.13).

In the end, a simple example of a consistent theory of pure quantum gravity is the one with the Lagrangian

$$
\begin{aligned}
\mathcal{L}= & -\frac{\sqrt{-g}}{2 \kappa^{2}} \mu^{-\varepsilon}\left[\frac{\gamma^{2}}{2} R-\frac{\gamma}{M^{2}}\left(R_{\mu \nu} R^{\mu \nu}-\frac{1}{2} R^{2}\right)-\frac{1}{M^{4}}\left(D_{\rho} R_{\mu \nu}\right)\left(D^{\rho} R^{\mu \nu}\right)+\frac{1}{2 M^{4}}\left(D_{\rho} R\right)\left(D^{\rho} R\right)\right. \\
& \left.+\frac{1}{M^{4}}\left(\alpha_{1}^{*} R_{\mu \nu} R^{\mu \rho} R_{\rho}^{\nu}+\alpha_{2}^{*} R R_{\mu \nu} R^{\mu \nu}+\frac{1}{72}\left(8+9 \alpha_{1}^{*}\right) R^{3}\right)+\mathcal{O}(\bar{\alpha})\right],
\end{aligned}
$$

where $\gamma$ is negative but arbitrary, $\alpha_{1,2}^{*}$ are the values listed in (2.13) and the corrections $\mathcal{O}(\bar{\alpha})$ are determined as explained above.

Now we repeat the analysis relaxing the simplifying condition $\eta=0$, i.e. we just assume $\xi=\alpha_{6}=0$ and study the conditions under which the cosmological constant $\lambda_{C}$ vanishes identically. The theory is no longer finite, because the parameters $\zeta, \gamma$ and $\eta$ can run.

The renormalization group consistency conditions generated by $\lambda_{C}=0$ give, at $\bar{\alpha}=0$,

$$
\begin{aligned}
& \zeta=\frac{1}{2} \gamma^{2}-\frac{1}{2} \eta \gamma+\frac{3}{4} \eta^{2}, \quad \gamma=-2 \eta \frac{\sigma+a_{\gamma}-3 a_{\eta}}{\tau-4 a_{\gamma}+2 a_{\eta}}, \\
& a_{\gamma}\left(\tau-4 a_{\gamma}+2 a_{\eta}\right)+2 a_{\eta}\left(\sigma+a_{\gamma}-3 a_{\eta}\right)=0 .
\end{aligned}
$$

An example of acceptable solution is (rounding to four decimal places)

$$
\zeta=43.6118 \eta^{2}, \quad \gamma=-8.7722 \eta, \quad \alpha_{3}=-0.0367,
$$

together with $\lambda_{C}=\xi=\alpha_{1}=\alpha_{2}=\alpha_{4}=\alpha_{5}=\alpha_{6}=0$. It is easy to check that the unitarity bounds (2.6) are satisfied if $\eta>0$. Moreover, the solutions can be extended to $\bar{\alpha} \neq 0$. The $\eta$ running is given by $\beta_{\eta}=0.7182 \bar{\alpha}$ and the runnings of $\zeta$ and $\gamma$ follow from their relations with $\zeta$.

Once we have a model where the cosmological constant vanishes identically, the asymptotic states and the $S$ matrix can be defined in the usual way. Then, the proof of perturbative unitarity can be worked out by combining the strategy of [14] (to show that the Lee-Wick poles do not propagate through the cuts in the cutting equations), with the strategy of ref. [5] (to show that the gauge degrees of freedom - i.e. those propagated by the gauge-dependent poles of $h_{\mu \nu}$ and the Faddeev-Popov ghosts - also do not propagate through the cuts). In particular, we must work in a gauge that interpolates between the 
Coulomb one and the one we used to derive the propagators (2.4) and (2.5). Artificial masses $m_{g}$ for the graviton are introduced to have control on the infrared divergences. When $m_{g} \neq 0$ the gauge degrees of freedom drop out by approaching the Coulomb limit arbitrarily without reaching it. After that, the limit $m_{g} \rightarrow 0$ can be safely taken in suitable combinations of amplitudes where the infrared divergences mutually cancel out. For details, see ref. [5].

\section{Coupling to matter}

Now we generalize the analysis of the previous section to the coupling to matter. In this context, "matter" refers to every classical field but the graviton, including gauge vectors. To begin with, we assume that the matter fields are massless and switch off all the matter self interactions. In other words, we take free massless scalars, fermions and vectors, and covariantize their actions to couple them to gravity. The matter Lagrangian $\mathcal{L}_{m}$ is given by

$$
\frac{\mathcal{L}_{m}}{\sqrt{-g}}=-\frac{1}{4} F_{\mu \nu} F^{\mu \nu}+i \bar{\psi} e_{a}^{\mu} \gamma^{a} D_{\mu} \psi+\frac{1}{2}\left(\partial_{\mu} \varphi\right) g^{\mu \nu}\left(\partial_{\nu} \varphi\right)+\frac{1}{12}(1+2 \varpi) R \varphi^{2}
$$

and the counterterms are [49-51]

$$
\frac{\mathcal{L}_{m \text { count }}}{\sqrt{-g}}=\frac{\mu^{-\varepsilon}}{(4 \pi)^{2} \varepsilon}\left[-2 c\left(R_{\mu \nu} R^{\mu \nu}-\frac{1}{3} R^{2}\right)-\frac{n_{s} \varpi^{2}}{18} R^{2}\right]
$$

where $c$ is known as "central charge" in conformal field theory, equal to

$$
c=\frac{1}{120}\left(n_{s}+6 n_{f}+12 n_{v}\right) .
$$

Here $n_{s}$ denotes the number of real scalar fields, $n_{f}$ is the number of Dirac fermions plus one half the number of Weyl fermions, while $n_{v}$ is the number of vector fields. Note that the right-hand side of (3.1) is proportional to the square of the Weyl tensor at $\varpi=0$, the reason being that the matter action is Weyl invariant in that case.

The counterterms (3.1) only affect the beta functions $\beta_{\gamma}$ and $\beta_{\eta}$. The corrected beta functions are obtained by making the replacements

$$
a_{\gamma} \rightarrow a_{\gamma}+2 c, \quad a_{\eta} \rightarrow a_{\eta}+\frac{2}{3} c+\frac{n_{s} \varpi^{2}}{9} .
$$

In the end, we have

$$
\begin{aligned}
& \beta_{\zeta}=-\frac{1}{2} \gamma \tau \bar{\alpha}-\eta \sigma \bar{\alpha}-4 z_{1} \bar{\alpha}^{2}, \quad \beta_{\gamma}=-2\left(a_{\gamma}+2 c\right) \bar{\alpha}, \quad \beta_{\eta}=-2\left(a_{\eta}+\frac{2}{3} c\right) \bar{\alpha}-\frac{2 n_{s} \varpi^{2}}{9} \bar{\alpha}, \\
& \beta_{C}=-\frac{3}{2}\left(4 \zeta-2 \gamma^{2}+2 \eta \gamma-3 \eta^{2}\right) \bar{\alpha}-2\left(2 u_{1} \gamma+2 v_{1} \eta+3 w_{1} \bar{\alpha}\right) \bar{\alpha}^{2}, \quad \beta_{\mathrm{QG}}=0 .
\end{aligned}
$$

where the higher-loop contributions need not coincide with those of the pure theory.

We inquire when we can prove perturbative unitarity again. In the simple case $\lambda_{C}=\eta=\xi=\alpha_{6}=0$, we impose (2.8) and the consistency conditions that follow from 
renormalization group invariance. As before, the condition $\beta_{C}=0$ can be solved immediately, since it just gives $\zeta$ in terms of the other parameters. The consistency conditions that make the solution renormalization group invariant can be obtained by making the replacements (3.2) inside (2.12). The net result is again that the theory must be completely finite. We must solve

$$
a_{\gamma}+2 c=0, \quad a_{\eta}+\frac{2}{3} c=-\frac{n_{s} \varpi^{2}}{9}, \quad \gamma \tau=-8 z_{1} \bar{\alpha} .
$$

Various acceptable solutions exist, with the matter content of the standard model $\left(n_{s}=4\right.$, $\left.n_{f}=45 / 2, n_{v}=12\right)$. An example of unitary quantum gravity coupled to massless nonself-interacting matter is the model which at $\bar{\alpha}=0$ has parameters

$$
\begin{aligned}
\zeta & =\frac{\gamma^{2}}{2}, \quad \alpha_{1}=8\left(\alpha_{3}-\frac{1}{9}\right), \quad \alpha_{2}=-3.3306, \quad \alpha_{3}=0.5721, \\
\lambda_{C} & =\eta=\varpi=\xi=\alpha_{4}=\alpha_{5}=\alpha_{6}=0,
\end{aligned}
$$

while the corrections for $\bar{\alpha} \neq 0$ are determined with the procedure explained before.

At very large distances, the standard model loses the QCD sector, the massive vector bosons as well as all the other massive particles. Only the free photon survives. If we couple it to the quantum gravity theory $(2.1)$, we obtain $\left(n_{s}=n_{f}=0, n_{v}=1\right)$ for example the solution

$$
\begin{aligned}
\zeta & =\frac{\gamma^{2}}{2}, \quad \alpha_{1}=8\left(\alpha_{3}-\frac{1}{9}\right), \quad \alpha_{2}=-3.8957, \quad \alpha_{3}=0.6716, \\
\lambda_{C} & =\eta=\varpi=\xi=\alpha_{4}=\alpha_{5}=\alpha_{6}=0 .
\end{aligned}
$$

When we add the masses and the matter self interactions, the beta functions $\beta_{C}$ receives nontrivial corrections. In general, setting it to zero and imposing the consistency conditions required by renormalization group invariance leads to a finite theory.

Call the couplings of the theory $\lambda_{i}$ and denote their beta functions by $\beta_{i}$. Assume we want to set some function $f(\lambda)$ of the couplings to zero. Then we must also set the beta function of $f(\lambda)$ to zero, and the beta function of the beta function, etc. We get the system of equations

$$
\begin{aligned}
f=0, \quad \beta_{i} f_{i}=0, \quad \beta_{j}\left(\beta_{i j} f_{i}+\beta_{i} f_{i j}\right) & =0, \\
\beta_{k}\left(\beta_{j k} \beta_{i j} f_{i}+\beta_{j} \beta_{i j k} f_{i}+3 \beta_{j} \beta_{i j} f_{i k}+\beta_{j} \beta_{i} f_{i j k}\right) & =0,
\end{aligned}
$$

etc., where $\beta_{i j_{1} \cdots j_{n}} \equiv \partial \beta_{i} /\left(\partial \lambda_{j_{1}} \cdots \partial \lambda_{j_{n}}\right)$ and $f_{j_{1} \cdots j_{n}} \equiv \partial f /\left(\partial \lambda_{j_{1}} \cdots \partial \lambda_{j_{n}}\right)$. We call the conditions (3.5) the renormalization group $(R G)$ chain generated by $f$. In our cases, the unitarity chain is the one starting from $f(\lambda)=\lambda_{C}$. In general, the RG chain is made of independent equations for the parameters $\lambda_{i}$ and so fixes all of them to constant values (assuming that such values do exist and are physically acceptable), which means that the theory is finite. When the beta functions are particularly simple, there may be exceptions where some parameters run after imposing the RG chain, as shown in the example (2.14).

In realistic models, the cosmological constant does not vanish identically, so we cannot prove perturbative unitarity in a strict sense. It might be possible to prove (a generalized 
notion of) perturbative unitarity in an unconventional approach, but we do not know this for sure at present. The other option is that unitarity is anomalous in the universe and the cosmological constant is the measure of such an anomaly.

It is worth noting that, under some assumptions, the proof of the cutting equations formally works even when the cosmological constant $\lambda_{C}$ is negative, because in that case the propagator acquires a sort of mass term. Although flat space is no longer a solution of the classical field equations, we can still expand around it, since the physics does not depend on the expansion we choose. Consider again the simple case $\eta=\xi=0$. Using the same gauge fixing as we used at $\lambda_{C}=0$, the graviton propagator becomes (2.4). Defining $x=p^{2} / M^{2}$, "unitarity" requires that the polynomial $2 \lambda_{C}+\zeta x+\gamma x^{2}+x^{3}$ that appears in the denominator have the form $(x-a)\left((x-b)^{2}+c\right)$ with $a \geqslant 0, b \geqslant 0, c>0$. Necessary conditions are $2 \lambda_{C}=-a\left(b^{2}+c\right)<0$, as well as $\zeta>0, \gamma<0$. The simplest case where such $a, b, c$ exist is when the parameters also satisfy $\gamma \zeta=2 \lambda_{C}$. Then, we have $a=-2 \lambda_{C} / \zeta$, $b=0, c=\zeta$. When $\gamma \zeta \leqslant 2 \lambda_{C}, b$ is positive and the solution continues to exist as long as $c$ stays positive. In these cases, the derivation of the cutting equations formally extends to the case of nonvanishing cosmological constant. Moreover, the examples of finite theories considered so far remain finite even when $\lambda_{C} \neq 0$, since the beta functions receive no contributions from the cosmological constant.

\section{The problem of uniqueness}

The theory with Lagrangian (2.1) is the simplest model belonging to the class of superrenormalizable theories of quantum gravity. The other models can be obtained from (2.1) by adding more and more higher derivatives and fulfilling the constraints due to unitarity and renormalizability by power counting. The Lagrangians are

$$
-2 \kappa^{2} \mu \frac{\mathcal{L}_{\mathrm{QG}}^{\prime}}{\sqrt{-g}}=2 \lambda_{C} M^{2}+\zeta R+\frac{1}{M^{2}} R_{\mu \nu} P_{n}\left(\square_{c} / M^{2}\right) R^{\mu \nu}-\frac{1}{2 M^{2}} R Q_{n}\left(\square_{c} / M^{2}\right) R+V(R),
$$

where $\square_{c}$ denotes the covariant D'Alembertian, $P_{n}, Q_{n}$ are real polynomials of degree $n>1$ and $V(R)$ is a linear combination of scalars that have dimensions ranging from 6 to $2 n+4$ and are built with at least three Riemann tensors (or their covariant derivatives).

In the extended class, it is easier to set the cosmological constant to zero at all energies. For example, for $V(R)=0, n>2$ there are only one-loop counterterms and the beta function $\beta_{C}$ of the cosmological constant is a linear combination of the coefficients $\zeta_{1}$ and $\zeta_{2}$ of the terms $R_{\mu \nu} \square_{c}^{n-2} R^{\mu \nu}$ and $R \square_{c}^{n-2} R$, plus a quadratic polynomial in the coefficients $\gamma$ and $\eta$ of $R_{\mu \nu} \square_{c}^{n-1} R^{\mu \nu}$ and $R \square_{c}^{n-1} R$. Moreover, $\zeta_{1}, \zeta_{2}, \gamma$ and $\eta$ do not run. Setting $\beta_{C}=0$ gives a relation between such constants, which can be easily solved for $\zeta_{1}$ or $\zeta_{2}$ in terms of the other three. The RG chain stops immediately. If the denominators of the propagators have the right form, the theory is also unitary. The beta function $\beta_{\zeta}$ is linear in $\gamma$ and $\eta$. The couplings of the quadratic terms $R_{\mu \nu} R^{\mu \nu}$ and $R^{2}$ may also have nontrivial beta functions.

In the end, the superrenormalizable models of quantum gravity that are unitary are infinitely many, which leads to a lack of uniqueness. The theory (2.1) is singled out among 
the others if we accept a sort of "minimum principle", stating that the right theory is just the simplest one. However, it would be better to have a really unique answer.

A possibility would be a theory with a dimensionless gauge coupling, that is to say a strictly renormalizable theory, which would make quantum gravity more similar to the other gauge theories. A conventional strictly renormalizable Lee-Wick model of quantum gravity in four dimensions does not exist, because the propagators would not have the structure (2.3). For this reason, we need an improved approach to the problem.

\section{$5 \quad$ Fake degrees of freedom}

In this section we investigate the idea of doubling the ghost poles of the free propagators and turn them into LW poles. An extra, fictitious LW scale $\mathcal{E}$ is introduced and removed at the end. This leads to a new quantization prescription. In the next section we explore the consequences of this idea in quantum gravity.

Start from the (massless) $\varphi^{4}$ scalar field theory

$$
\mathcal{L}=\frac{1}{2}\left(\partial_{\mu} \varphi\right)\left(\partial^{\mu} \varphi\right)-\frac{\lambda}{4 !} \varphi^{4}
$$

in four dimensions and formulate it in Euclidean space. We write the Euclidean propagator $1 / p_{E}^{2}$ as $p_{E}^{2} /\left(p_{E}^{2}\right)^{2}$, where $p_{E}$ is the Euclidean momentum. Then, we deform the propagator with the help of the fictitious LW scale $\mathcal{E}$ into

$$
\frac{p_{E}^{2}}{\left(p_{E}^{2}\right)^{2}+\mathcal{E}^{4}}
$$

and consider the limit $\mathcal{E} \rightarrow 0$. If we first let $\mathcal{E}$ tend to zero and then Wick rotate, we obtain the usual scalar field theory. On the other hand, if we first Wick rotate, then let the scale $\mathcal{E}$ tend to zero, the $S$ matrix is identically one. Indeed, at $\mathcal{E}>0$ we obtain a Lee-Wick model. Formulated as a nonanalytically Wick rotated Euclidean theory, it is perturbatively unitary and has no physical degree of freedom.

We point out that the prescription we have just defined does not give the principal value of $-1 / p^{2}$ after the Wick rotation. Indeed, since we are coming from the Euclidean space, the poles $p^{0}=\sqrt{\mathbf{p}^{2} \pm i \mathcal{E}^{2}}$ are located below the integration path, while the poles $p^{0}=-\sqrt{\mathbf{p}^{2} \pm i \mathcal{E}^{2}}$ are located above. Instead, the principal value places the poles $p^{0}=$ $\pm \sqrt{\mathbf{p}^{2} \pm i \mathcal{E}^{2}}$ above the integration path and the poles $p^{0}=\mp \sqrt{\mathbf{p}^{2} \pm i \mathcal{E}^{2}}$ below.

For this reason, our construction defines a distribution of a new type. Flipping the overall sign, after the Wick rotation we write it as

$$
\lim _{\mathcal{E} \rightarrow 0} \frac{p^{2}}{\left[\left(p^{2}\right)^{2}+\mathcal{E}^{4}\right]_{\mathrm{LW}}} .
$$

The subscript "LW" in the denominator is to remind us about the positions of the poles with respect to the integration path (the right pair of poles being placed below and the left pair being placed above).

A good check that (5.2) is well defined can be made by calculating the bubble diagram explicitly with the technique explained in ref. [11] and then take the limit $\mathcal{E} \rightarrow 0$. The 
calculation can be carried out to the very end and, after renormalizing the ultraviolet divergence, gives for $p$ real

$$
-\frac{i}{4(4 \pi)^{2}} \ln \frac{\left(p^{2}\right)^{2}}{\mu^{4}}
$$

where we have included the combinatorial factor $1 / 2$. The complex energy plane is divided into three disjoint regions. The main region $\mathcal{A}_{0}$ is the one that contains the imaginary axis, where the Wick rotation is analytic. The other two regions $\mathcal{A}_{1}$ and $\mathcal{A}_{1}^{\prime}$ are symmetric with respect to the imaginary axis and intersect the real axis in the half lines $p^{0}>|\boldsymbol{p}|$ and $p^{0}<-|\boldsymbol{p}|$. At $\boldsymbol{p} \neq 0$ the half $p^{0}$ plane with $\operatorname{Re}\left[p^{0}\right] \geqslant 0$ looks like

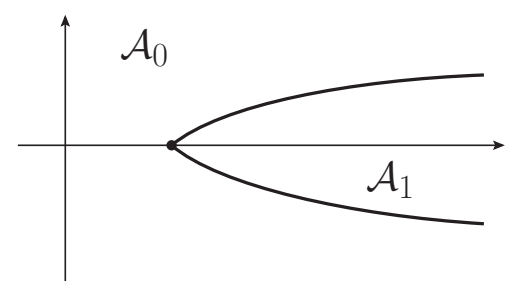

The boundary separating the regions $\mathcal{A}_{0}$ and $\mathcal{A}_{1}$ can be deformed arbitrarily, as long as it does not intersect the real axis anywhere but in the threshold $p^{0}=|\boldsymbol{p}|$. The real part of the diagram vanishes for all real values of $p$, which confirms that the $S$ matrix is identically 1. We may say that (5.2) turns the degree of freedom of the $\varphi^{4}$ theory into a fake degree of freedom.

Let us compare this result with the one given by the Feynman prescription, which is

$$
-\frac{i}{2(4 \pi)^{2}} \ln \frac{-p^{2}-i \epsilon}{\mu^{2}}
$$

The two expressions (5.3) and (5.5) coincide in the main region $\mathcal{A}_{0}$. However, the Feynman prescription does not predict the region $\mathcal{A}_{1}$, but maximally extends $\mathcal{A}_{0}$, so the amplitude (5.5) has a discontinuity on the real axis above the threshold $p^{0}=|\boldsymbol{p}|$.

With a similar procedure, we can turn the ghosts into fake degrees of freedom. Consider the higher-derivative theory with Lagrangian

$$
\mathcal{L}=\frac{1}{2}\left(\partial_{\mu} \varphi\right)\left(\zeta-\gamma \frac{\square}{M^{2}}\right)\left(\partial^{\mu} \varphi\right)-\frac{\lambda}{4 !} \varphi^{4}
$$

with $\zeta>0, \gamma<0$. The Euclidean propagator is

$$
\frac{M^{2}}{p_{E}^{2}\left(\zeta M^{2}-\gamma p_{E}^{2}\right)}=\frac{1}{\zeta p_{E}^{2}}+\frac{\gamma}{\zeta\left(\zeta M^{2}-\gamma p_{E}^{2}\right)}
$$

and its naive Wick rotation to Minkowski spacetime propagates a physical massless scalar and a massive ghost. Let us deform (5.6) into

$$
\frac{1}{\zeta p_{E}^{2}}+\frac{\gamma\left(\zeta M^{2}-\gamma p_{E}^{2}\right)}{\zeta\left[\left(\zeta M^{2}-\gamma p_{E}^{2}\right)^{2}+\mathcal{E}^{4}\right]}
$$


After the Wick rotation, we find (multiplying by $-i$ )

$$
\frac{i}{\zeta\left(p^{2}+i \epsilon\right)}-\frac{i \gamma\left(\zeta M^{2}+\gamma p^{2}\right)}{\zeta\left[\left(\zeta M^{2}+\gamma p^{2}\right)^{2}+\mathcal{E}^{4}\right]_{\mathrm{LW}}},
$$

which just propagates a massless particle, since the poles at $p^{2}=-\left(\zeta M^{2} \pm i \mathcal{E}^{2}\right) / \gamma$ compensate each other in the cut propagators for every $\mathcal{E}>0$, then also for $\mathcal{E} \rightarrow 0$.

The procedure is very general and can be used to make sense of higher-derivative quantum gravity, by turning its ghosts into fakes, as we explain in the next section.

We have said in section 2 that the renormalization of the theory at $\mathcal{E} \neq 0$ coincides with the renormalization of its Euclidean version. It is easy to show that this property survives the limit $\mathcal{E} \rightarrow 0$. We know that the complex energy plane is divided into disjoint regions at $\mathcal{E} \neq 0$, the main one being the region that contains the imaginary axis, where the Wick rotation is analytic. The other regions are related to the main one by means of a nonanalytic procedure. Renormalizability holds because the divergent parts of the amplitudes just concern the main region. In the limit $\mathcal{E} \rightarrow 0$ the intersection between the main region and the real axis is the interval $-|\mathbf{p}|<p^{0}<|\mathbf{p}|$.

For example, in the case of the bubble diagram, two LW pinchings have thresholds on the real axis, their threshold being $p^{2}=2 \mathcal{E}^{2}$. The condition of pinching is

$$
\left|p^{0}\right|=\sqrt{\mathbf{k}^{2}+i \mathcal{E}^{2}}+\sqrt{(\mathbf{k}-\mathbf{p})^{2}-i \mathcal{E}^{2}},
$$

where $p$ is the external momentum and $k$ is the loop momentum. This condition cannot be solved for arbitrarily large loop space momentum $\mathbf{k}$. Therefore, the ultraviolet divergences are not affected by the LW pinching. The conclusion also holds at $\mathcal{E} \rightarrow 0$, where the condition becomes

$$
\left|p^{0}\right|=|\mathbf{k}|+|\mathbf{k}-\mathbf{p}|,
$$

which is also bounded in $|\mathbf{k}|$ when the external momentum is fixed.

We can use the new distribution (5.2) to build theories with unforeseen properties. For example, consider the theory with Lagrangian

$$
\mathcal{L}=\frac{1}{2}\left(\partial_{\mu} \varphi\right)\left(\partial^{\mu} \varphi\right)+\frac{1}{2}\left(\partial_{\mu} \chi\right)\left(\partial^{\mu} \chi\right)-\frac{\lambda}{4 !} \varphi^{4}-\frac{\lambda^{\prime}}{4 !} \chi^{4}-\frac{\lambda^{\prime \prime}}{4} \varphi^{2} \chi^{2}
$$

and endow the scalar $\varphi$ with the usual prescription and $\chi$ with the prescription (5.2). So doing, $\chi$ does not contribute to the cuts, nor to the initial and final states. However, it does contribute to the loop diagrams. If we integrate $\chi$ out, we obtain an effective renormalizable, unitary, nonlocal theory of the self-interacting scalar field $\varphi$.

\section{Quantum gravity with a dimensionless gauge coupling}

In this section, we consider the theory described by the Lagrangian

$$
-2 \kappa^{2} \frac{\mathcal{L}_{\mathrm{QG}}}{\sqrt{-g}}=2 \Lambda_{C}+\zeta R-\frac{\gamma}{M^{2}} R_{\mu \nu} R^{\mu \nu}+\frac{1}{2 M^{2}}(\gamma-\eta) R^{2},
$$

with $\zeta>0$ and $\gamma<0$. Although the form of $\mathcal{L}_{\mathrm{QG}}$ coincides with the one of the well-known theory of refs. [6-9], we want to quantize it in a new way. 
For the time being, we neglect the cosmological constant. At $\eta=0$ the graviton propagator reads

$$
\left\langle h_{\mu \nu}(p) h_{\rho \sigma}(-p)\right\rangle_{\eta=0}^{\text {free }}=\frac{i M^{2}}{2 p^{2}\left(\zeta M^{2}+\gamma p^{2}\right)}\left(\eta_{\mu \rho} \eta_{\nu \sigma}+\eta_{\mu \sigma} \eta_{\nu \rho}-\eta_{\mu \nu} \eta_{\rho \sigma}\right) .
$$

To have perturbative unitarity in this case, we proceed as explained in the previous section, which means that we convert (6.2) into

$$
\left\{\frac{1}{p^{2}+i \epsilon}-\frac{\gamma\left(\zeta M^{2}+\gamma p^{2}\right)}{\left[\left(\zeta M^{2}+\gamma p^{2}\right)^{2}+\mathcal{E}^{4}\right]_{\mathrm{LW}}}\right\} \frac{i}{2 \zeta}\left(\eta_{\mu \rho} \eta_{\nu \sigma}+\eta_{\mu \sigma} \eta_{\nu \rho}-\eta_{\mu \nu} \eta_{\rho \sigma}\right),
$$

where $\mathcal{E}$ is the fake LW scale, which must tend to zero pretty much like the width $\epsilon$. Similarly, at $\eta \neq 0$ we find

$$
\left\langle h_{\mu \nu}(p) h_{\rho \sigma}(-p)\right\rangle_{\eta}^{\text {free }}=\left\langle h_{\mu \nu}(p) h_{\rho \sigma}(-p)\right\rangle_{\eta=0}^{\text {free }}-\frac{i \eta M^{2}}{2\left(p^{2}\right)^{2}} \frac{\left(p^{2} \eta_{\mu \nu}+2 p_{\mu} p_{\nu}\right)\left(p^{2} \eta_{\rho \sigma}+2 p_{\rho} p_{\sigma}\right)}{\left(\zeta M^{2}+\gamma p^{2}\right)\left[\zeta M^{2}+(\gamma-3 \eta) p^{2}\right]} .
$$

Assuming that $\gamma<3 \eta$, the extra contribution can be cured by turning (6.3) into

$$
-\frac{i \eta}{2} \frac{\left(p^{2} \eta_{\mu \nu}+2 p_{\mu} p_{\nu}\right)\left(p^{2} \eta_{\rho \sigma}+2 p_{\rho} p_{\sigma}\right)}{\left[\left(p^{2}\right)^{2}+\mathcal{E}^{4}\right]_{\mathrm{LW}}} \frac{M^{2}\left(\zeta M^{2}+\gamma p^{2}\right)\left(\zeta M^{2}+(\gamma-3 \eta) p^{2}\right)}{\left[\left(\zeta M^{2}+\gamma p^{2}\right)^{2}+\mathcal{E}^{4}\right]_{\mathrm{LW}}\left[\left(\zeta M^{2}+(\gamma-3 \eta) p^{2}\right)^{2}+\mathcal{E}^{4}\right]_{\mathrm{LW}}} .
$$

A similar procedure must be applied to the propagators of the Faddeev-Popov ghosts.

The theory (6.1) is the unique renormalizable higher-derivative theory of quantum gravity whose gauge coupling $\tilde{\kappa}=\kappa M$ is dimensionless with respect to the high-energy power counting. Indeed, if we define $\tilde{h}_{\mu \nu}=h_{\mu \nu} / M$ and expand the metric tensor as $g_{\mu \nu}=\eta_{\mu \nu}+2 \tilde{\kappa} \tilde{h}_{\mu \nu}$, the Lagrangian $\mathcal{L}_{\mathrm{QG}}$ behaves like $\sim(\square \tilde{h})^{2}$ at high energies, times dimensionless constants. With the prescriptions just given, the theory is also perturbatively unitary up to corrections due to the cosmological constant.

We know that in general the cosmological constant is turned on by the radiative corrections, which prevents us from proving perturbative unitarity in a strict sense. Modified models with an identically vanishing cosmological constant might exist. For example, it is likely possible to build supersymmetric extensions of the theory (6.1) that have one-loop exact beta functions or are even finite, because similar constructions are familiar in supersymmetric theories of fields of spins $0,1 / 2$ and 1 [52]. If such models are finite, the cosmological constant can be switched off at no cost. If they have one-loop exact beta functions, extra conditions have to be imposed in order to fulfill the renormalization group chain (3.5) that follows from $\Lambda_{C}=0$. Probably, the conditions are simple enough to admit nontrivial solutions, as we found in sections 2 and 3 for the superrenormalizable theory (2.1) and its coupling to matter. In all such cases, we may provide examples of theories quantum gravity (coupled to matter) with a dimensionless gauge coupling and an identically vanishing cosmological constant, where the proof of unitarity can be carried out to the very end. However, it is unlikely that the ultimate theory of nature will have an identically vanishing cosmological constant, so we must be prepared to accept that there may be a small unitarity anomaly in the universe. 


\section{Conclusions}

In this paper we have studied the main options for a consistent, local quantum field theory of the gravitational interactions. Superrenormalizable higher-derivatives theories of gravity can be built as Lee-Wick models and formulated as nonanalytically Wick rotated Euclidean theories. They are perturbatively unitary when the cosmological constant vanishes. The simplest example is encoded in formula (2.1), provided the parameters satisfy suitable restrictions. The other models of this class can be build by adding more higher-derivatives and fulfilling the Lee-Wick unitarity conditions. The possibilities are infinitely many, which raises the question of uniqueness.

A better possibility is the theory (6.1), because it has a dimensionless gauge constant, which makes it unique and more similar to the gauge theories that describe the other interactions of nature. The Lagrangian (6.1) cannot be quantized in the conventional LeeWick way. This lead us to introduce a new concept, the fake degrees of freedom, and a prescription different from the usual one. Taking advantage of the Lee-Wick idea, a ghost (or a normal degree of freedom) can be turned into a fake degree of freedom, which does not contribute to the physical spectrum and does not propagate through the cuts of the cutting equations. So doing, the ghosts of higher-derivative gravity can be eliminated.

The renormalization of the theory (6.1) is obviously richer than the one of a superrenormalizable theory like (2.1), because nontrivial radiative corrections to the beta functions and the anomalous dimensions are expected to all orders. Again, this is similar to what we know from the other gauge theories that successfully describe nature.

If we accept that the gauge couplings are dimensionless, then there is only one theory of the four interactions of nature, made of the gauge sector of the standard model coupled to the quantum gravity theory (6.1). Constraining the matter sector is obviously harder.

In several models it is possible to turn the cosmological constant off to all energies, consistently with the renormalization group. However, the more realistic models have a nonvanishing cosmological constant, which might be the signal of a small unitarity anomaly.

It can be interesting to study the phenomenological implications of the theory (6.1) coupled to the standard model. Some arguments existing in the literature (see for example ref. [53]) might survive after switching to the correct formulation of the theory, others might have to be reconsidered.

\section{Acknowledgments}

We are grateful to U. Aglietti and M. Piva for useful discussions.

Open Access. This article is distributed under the terms of the Creative Commons Attribution License (CC-BY 4.0), which permits any use, distribution and reproduction in any medium, provided the original author(s) and source are credited. 


\section{References}

[1] G. 't Hooft and M. Veltman, One-loop divergences in the theory of gravitation, Ann. Inst. H. Poincaré Phys. Theor. A 20 (1974) 69.

[2] P. Van Nieuwenhuizen, On the renormalization of quantum gravitation without matter, Annals Phys. 104 (1977) 197 [INSPIRE].

[3] M.H. Goroff and A. Sagnotti, The ultraviolet behavior of Einstein gravity, Nucl. Phys. B 266 (1986) 709 [InSPIRE].

[4] A.E.M. van de Ven, Two loop quantum gravity, Nucl. Phys. B 378 (1992) 309 [InSPIRE].

[5] D. Anselmi, Aspects of perturbative unitarity, Phys. Rev. D 94 (2016) 025028 [http://renormalization.com/16a1/] [arXiv:1606.06348] [INSPIRE].

[6] K.S. Stelle, Renormalization of higher derivative quantum gravity, Phys. Rev. D 16 (1977) 953 [INSPIRE].

[7] J. Julve and M. Tonin, Quantum gravity with higher derivative terms, Nuovo Cim. B 46 (1978) 137 [INSPIRE].

[8] E.S. Fradkin and A.A. Tseytlin, Renormalizable asymptotically free quantum theory of gravity, Nucl. Phys. B 201 (1982) 469 [InSPIRE].

[9] I.G. Avramidi and A.O. Barvinsky, Asymptotic freedom in higher derivative quantum gravity, Phys. Lett. B 159 (1985) 269 [INSPIRE].

[10] U.G. Aglietti and D. Anselmi, Inconsistency of Minkowski higher-derivative theories, Eur. Phys. J. C 77 (2017) 84 [http://renormalization.com/16a2/] [arXiv:1612.06510] [INSPIRE].

[11] D. Anselmi and M. Piva, A new formulation of Lee-Wick quantum field theory, [http://renormalization.com/17a1/] arXiv: 1703.04584 [INSPIRE].

[12] T.D. Lee and G.C. Wick, Negative metric and the unitarity of the S-matrix, Nucl. Phys. B 9 (1969) 209 [inSPIRE].

[13] T.D. Lee and G.C. Wick, Finite theory of quantum electrodynamics, Phys. Rev. D 2 (1970) 1033 [InSPIRE].

[14] D. Anselmi and M. Piva, Perturbative unitarity of Lee-Wick quantum field theory, http://renormalization.com/17a2/ [arXiv:1703.05563] [INSPIRE].

[15] R.E. Cutkosky, P.V. Landshoff, D.I. Olive and J.C. Polkinghorne, A non-analytic S matrix, Nucl. Phys. B 12 (1969) 281 [InSPIRE].

[16] B. Grinstein, D. O'Connell and M.B. Wise, Causality as an emergent macroscopic phenomenon: the Lee-Wick O(N) model, Phys. Rev. D 79 (2009) 105019 [arXiv:0805.2156] [INSPIRE].

[17] B. Grinstein, D. O'Connell and M.B. Wise, The Lee-Wick standard model, Phys. Rev. D 77 (2008) 025012 [arXiv:0704.1845] [INSPIRE].

[18] C.D. Carone and R.F. Lebed, Minimal Lee-Wick extension of the standard model, Phys. Lett. B 668 (2008) 221 [arXiv:0806.4555] [InSPIRE].

[19] J.R. Espinosa and B. Grinstein, Ultraviolet properties of the Higgs sector in the Lee-Wick standard model, Phys. Rev. D 83 (2011) 075019 [arXiv:1101.5538] [INSPIRE]. 
[20] C.D. Carone and R.F. Lebed, A higher-derivative Lee-Wick standard model, JHEP 01 (2009) 043 [arXiv:0811.4150] [INSPIRE].

[21] B. Grinstein and D. O'Connell, One-Loop Renormalization of Lee-Wick Gauge Theory, Phys. Rev. D 78 (2008) 105005 [arXiv:0801.4034] [INSPIRE].

[22] C.D. Carone, Higher-Derivative Lee-Wick Unification, Phys. Lett. B 677 (2009) 306 [arXiv: 0904.2359] [INSPIRE].

[23] E. Tomboulis, $1 / N$ Expansion and Renormalization in Quantum Gravity, Phys. Lett. B 70 (1977) 361 [InSPIRE].

[24] E. Tomboulis, Renormalizability and Asymptotic Freedom in Quantum Gravity, Phys. Lett. B 97 (1980) 77 [INSPIRE].

[25] L. Modesto and I.L. Shapiro, Superrenormalizable quantum gravity with complex ghosts, Phys. Lett. B 755 (2016) 279 [arXiv:1512.07600] [InSPIRE].

[26] L. Modesto, Super-renormalizable or finite Lee-Wick quantum gravity, Nucl. Phys. B 909 (2016) 584 [arXiv:1602.02421] [InSPIRE].

[27] L. Modesto, L. Rachwal and I.L. Shapiro, Renormalization group in super-renormalizable quantum gravity, arXiv:1704.03988 [INSPIRE].

[28] S. Weinberg, Ultraviolet divergences in quantum theories of gravitation, in An Einstein centenary survey, S. Hawking and W. Israel eds., Cambridge University Press, Cambridge U.K. (1979), pg. 790.

[29] O. Lauscher and M. Reuter, Ultraviolet fixed point and generalized flow equation of quantum gravity, Phys. Rev. D 65 (2002) 025013 [hep-th/0108040] [INSPIRE].

[30] R. Percacci and D. Perini, Asymptotic safety of gravity coupled to matter, Phys. Rev. D 68 (2003) 044018 [hep-th/0304222] [INSPIRE].

[31] D.F. Litim, Fixed points of quantum gravity, Phys. Rev. Lett. 92 (2004) 201301 [hep-th/0312114] [INSPIRE].

[32] R. Percacci, A Short introduction to asymptotic safety, in Proceedings of "Time and matter" conference, Budva Montenegro (2010) [arXiv:1110.6389] [INSPIRE].

[33] Yu.V. Kuz'min, Finite nonlocal gravity, Sov. J. Nucl. Phys. 50 (1989) 6 [Yad. Fiz. 50 (1989) 1630].

[34] E.T. Tomboulis, Superrenormalizable gauge and gravitational theories, hep-th/9702146 [INSPIRE].

[35] L. Modesto, Super-renormalizable quantum gravity, Phys. Rev. D 86 (2012) 044005 [arXiv: 1107.2403] [INSPIRE].

[36] L. Modesto, Finite quantum gravity, arXiv:1305.6741 [INSPIRE].

[37] F. Briscese, L. Modesto and S. Tsujikawa, Super-renormalizable or finite completion of the Starobinsky theory, Phys. Rev. D 89 (2014) 024029 [arXiv: 1308.1413] [InSPIRE].

[38] T. Biswas, E. Gerwick, T. Koivisto and A. Mazumdar, Towards singularity and ghost free theories of gravity, Phys. Rev. Lett. 108 (2012) 031101 [arXiv:1110.5249] [INSPIRE].

[39] D. Chialva and A. Mazumdar, Cosmological implications of quantum corrections and higher-derivative extension, Mod. Phys. Lett. A 30 (2015) 1540008 [arXiv:1405.0513] [INSPIRE]. 
[40] R.E. Cutkosky, Singularities and discontinuities of Feynman amplitudes, J. Math. Phys. 1 (1960) 429 [INSPIRE].

[41] M.J.G. Veltman, Unitarity and causality in a renormalizable field theory with unstable particles, Physica 29 (1963) 186 [InSPIRE].

[42] G. 't Hooft, Renormalization of massless Yang-Mills fields, Nucl. Phys. B 33 (1971) 173 [INSPIRE].

[43] G. 't Hooft, Renormalizable Lagrangians for massive Yang-Mills fields, Nucl. Phys. B 35 (1971) 167 [inSPIRE].

[44] G. Narain and R. Anishetty, Short distance freedom of quantum gravity, Phys. Lett. B 711 (2012) 128 [arXiv:1109.3981] [InSPIRE].

[45] G. Narain and R. Anishetty, Unitary and renormalizable theory of higher derivative gravity, J. Phys. Conf. Ser. 405 (2012) 012024 [arXiv: 1210.0513] [InSPIRE].

[46] D. Anselmi, Properties of the classical action of quantum gravity, JHEP 05 (2013) 028 [http://renormalization.com/13a2/] [arXiv:1302.7100] [INSPIRE].

[47] S.B. Giddings, The Boundary $S$ matrix and the AdS to CFT dictionary, Phys. Rev. Lett. 83 (1999) 2707 [hep-th/9903048] [INSPIRE].

[48] V. Balasubramanian, S.B. Giddings and A.E. Lawrence, What do CFTs tell us about Anti-de Sitter space-times?, JHEP 03 (1999) 001 [hep-th/9902052] [INSPIRE].

[49] S.J. Hathrell, Trace anomalies and $\lambda \phi^{4}$ theory in curved space, Annals Phys. 139 (1982) 136 [INSPIRE].

[50] S.J. Hathrell, Trace anomalies and QED in curved space, Annals Phys. 142 (1982) 34 [INSPIRE].

[51] M.D. Freeman, The renormalization of non-Abelian gauge theories in curved space-time, Annals Phys. 153 (1984) 339 [INSPIRE].

[52] P.S. Howe, K.S. Stelle and P.C. West, A class of finite four-dimensional supersymmetric field theories, Phys. Lett. 124B (1983) 55 [INSPIRE].

[53] A. Salvio and A. Strumia, Agravity, JHEP 06 (2014) 080 [arXiv:1403.4226] [INSPIRE]. 